\title{
Maps on states preserving the relative entropy
}

\author{
Lajos Molnár ${ }^{a)}$ \\ Institute of Mathematics, University of Debrecen, P.O. Box 12, H-4010 Debrecen, \\ Hungary
}

(Received 20 November 2007; accepted 26 February 2008; published online 31 March 2008)

Let $H$ be a finite dimensional Hilbert space. The aim of this short note is to prove that every bijective map on the space $\mathcal{S}(H)$ of all density operators on $H$ which preserves the relative entropy is of the form $\phi(\rho)=U \rho U^{*}$ with some unitary or antiunitary operator $U$ on $H$. (C) 2008 American Institute of Physics.

[DOI: $10.1063 / 1.2898693]$

Quantum relative entropy is one of the most fundamental numerical quantities appearing in quantum information theory. It is the quantum analog of the relative entropy in classical information theory that provides a measure of distinguishability between quantum states. The general reference book of quantum relative entropy is Ref. 6. For recent reviews, see the papers in Refs. 7 and 8 and Chap. 12 in the book in Ref. 1.

In what follows, let $H$ be a finite dimensional Hilbert space. Denote by $\mathcal{S}(H)$ the space of all density operators (i.e., positive semidefinite operators with unit trace) on $H$. The elements of $\mathcal{S}(H)$ represent the states of the quantum systems to which the Hilbert space $H$ corresponds.

The relative entropy between the states represented by the density operators $\rho, \sigma \in \mathcal{S}(H)$ is defined by

$$
S(\rho \| \sigma)=\operatorname{tr} \rho(\log \rho-\log \sigma) .
$$

Here, tr stands for the usual trace functional. It is well known that the quantity $S(\rho \| \sigma)$ is always non-negative; it is finite if and only if $\operatorname{supp} \rho \subset \operatorname{supp} \sigma$ (supp standing for the orthogonal complement of the kernel of density operators), and it is zero if and only if $\rho=\sigma$.

It is apparent that the unitary and antiunitary transformations leave the relative entropy invariant (see, e.g., Ref. 1, p. 307). This means that if $U$ is a unitary or antiunitary operator on $H$, then we have

$$
S\left(U \rho U^{*} \| U \sigma U^{*}\right)=S(\rho \| \sigma)
$$

for every $\rho, \sigma \in \mathcal{S}(H) .{ }^{1}$ The aim of this short note is to prove the converse. Namely, in what follows, we show that any bijective transformation $\phi: \mathcal{S}(H) \rightarrow \mathcal{S}(H)$ which leaves the relative entropy invariant is necessarily of the form $\phi(\rho)=U \rho U^{*}[\rho \in \mathcal{S}(H)]$ with some unitary or antiunitary operator $U$ on $H$.

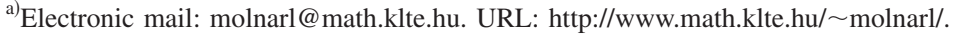

${ }^{1}$ According to the referee's wish, here is the sketch of the proof of this fact. First, $U \log \rho U^{*}=\log U \rho U^{*}$ holds for every density operator $\rho$. Using this, we compute

$$
U \rho U^{*}\left(\log U \rho U^{*}-\log U \sigma U^{*}\right)=U \rho U^{*}\left(U \log \rho U^{*}-U \log \sigma U^{*}\right)=U \rho(\log \rho-\log \sigma) U^{*} .
$$
}

However, for the trace of this latter operator, we have

$$
\operatorname{tr} U \rho(\log \rho-\log \sigma) U^{*}=\operatorname{tr} \rho(\log \rho-\log \sigma) U^{*} U=\operatorname{tr} \rho(\log \rho-\log \sigma)
$$

This gives the desired invariance property (1). 
To explain the content of our result, we recall Wigner's fundamental theorem on the form of symmetry transformations. A bijective map $\phi$ on the set of all rank-1 projections (pure states) on $H$ is called a symmetry transformation if it preserves the quantity tr $p q$ called the transition probability between pure states, i.e., if $\phi$ has the property that

$$
\operatorname{tr} \phi(p) \phi(q)=\operatorname{tr} p q
$$

holds for all rank-1 projections $p, q$ on $H$. It is clear that every transformation $\phi$ of the form $\phi(p)=U p U^{*}$ with some unitary or antiunitary operator $U$ on $H$ is a symmetry transformation. Wigner's theorem says that the converse statement is also true: every symmetry transformation can be obtained in that way. Hence, our present result can be considered as a Wigner-type result concerning state transformations leaving the relative entropy invariant. Similar results of the same spirit on state transformations preserving Uhlmann's fidelity, trace distance, Bures distance, or measure of compatibility can be found in the papers in Refs. 2, 4, and 5 (also see Secs. 2.3 and 2.4 in our recent book in Ref. 3).

In what follows, we formulate and prove the already announced result.

Theorem: Let $\phi: \mathcal{S}(H) \rightarrow \mathcal{S}(H)$ be a bijective map which preserves the relative entropy, i.e., which satisfies

$$
S(\phi(\rho) \| \phi(\sigma))=S(\rho \| \sigma)
$$

for every $\rho, \sigma \in \mathcal{S}(H)$. Then, there is an either unitary or antiunitary operator $U$ on $H$ such that $\phi$ is of the form

$$
\phi(\rho)=U \rho U^{*} \quad(\rho \in \mathcal{S}(H)) .
$$

Proof: As the relative entropy $S(\rho \| \sigma)$ is finite if and only if supp $\rho \subset \operatorname{supp} \sigma$, we deduce that $\phi$ has the property

$$
\operatorname{supp} \rho \subset \operatorname{supp} \sigma \Leftrightarrow \operatorname{supp} \phi(\rho) \subset \operatorname{supp} \phi(\sigma)
$$

for any $\rho, \sigma \in \mathcal{S}(H)$. As equality of sets can be expressed by two-sided inclusion, we infer that

$$
\operatorname{supp} \rho=\operatorname{supp} \sigma \Leftrightarrow \operatorname{supp} \phi(\rho)=\operatorname{supp} \phi(\sigma)
$$

and next that

$$
\operatorname{supp} \rho \varsubsetneqq \operatorname{supp} \sigma \Leftrightarrow \operatorname{supp} \phi(\rho) \varsubsetneqq \operatorname{supp} \phi(\sigma)
$$

for any $\rho, \sigma \in \mathcal{S}(H)$.

It is easy to see that the rank of an operator $\rho \in \mathcal{S}(H)$ is $n$ if and only if there is a chain

$$
\operatorname{supp} \rho_{1} \varsubsetneqq \operatorname{supp} \rho_{2} \varsubsetneqq \cdots \varsubsetneqq \operatorname{supp} \rho_{n} \subset \operatorname{supp} \rho
$$

of supports of length $n$ and there does not exist another such chain of length strictly larger than $n$. By the properties (2) and (3) of $\phi$, it now follows that $\phi$ preserves the rank of the elements of $\mathcal{S}(H)$. In particular, $\rho \in \mathcal{S}(H)$ is a rank-1 projection if and only if so is $\phi(\rho)$.

Let $\sigma \in \mathcal{S}(H)$ be a rank-2 operator of the form $\sigma=\lambda p+\mu q$, where $p, q \in \mathcal{S}(H)$ are mutually orthogonal rank-1 projections and $0<\lambda<\mu<1$ with $\lambda+\mu=1$. For any rank-1 projection $r$ on $H$, we easily have

$$
S(r \| \sigma)= \begin{cases}-[\log \lambda \operatorname{tr} r p+\log \mu \operatorname{tr} r q] & \text { if supp } r \subset \operatorname{supp} \sigma, \\ \infty & \text { otherwise. }\end{cases}
$$

As $r$ varies, the quantities $\operatorname{tr} r p$, tr $r q$ run through the set of all pairs of non-negative real numbers with sum 1. Consequently, the relative entropy $S(r \| \sigma)$ runs through the closed interval $[-\log \mu$, $-\log \lambda]$ plus the possible value $\infty$. This shows that from that set of entropies, we can recover the eigenvalues of the operator $\sigma$. Using this observation and the facts that $\phi$ preserves the relative 
entropy and $\phi(r)$ runs through the whole set of rank-1 projections, we deduce that the rank-2 operator $\phi(\sigma)$ is of the form

$$
\phi(\sigma)=\lambda p^{\prime}+\mu q^{\prime}
$$

with some mutually orthogonal rank-1 projections $p^{\prime}, q^{\prime}$ on $H$. Clearly, any element of a nontrivial compact real interval is a unique convex combination of the endpoints. Therefore, we have $S(r \| \sigma)=-\log \lambda$ if and only if $\operatorname{tr} r p=1$. It follows, for example, from the criterion of equality in the Cauchy-Schwarz inequality that tr $r p=1$ holds if and only if $r=p$. We now have the following chain of equivalences:

$$
r=p \Leftrightarrow S(r \| \sigma)=-\log \lambda \Leftrightarrow S(\phi(r) \| \phi(\sigma))=-\log \lambda \Leftrightarrow S\left(\phi(r) \| \lambda p^{\prime}+\mu q^{\prime}\right)=-\log \lambda \Leftrightarrow \phi(r)=p^{\prime},
$$

yielding $\phi(p)=p^{\prime}$. We similarly have $\phi(q)=q^{\prime}$. Therefore, we obtain

$$
\phi(\lambda p+\mu q)=\lambda \phi(p)+\mu \phi(q) .
$$

In particular, it follows that $\phi$ preserves the mutual orthogonality between rank-1 projections.

Let now $p, r$ be different rank-1 projections which are not orthogonal to each other. Pick a rank-1 projection $q$ which is orthogonal to $p$ and has the property that $\operatorname{supp} r \subset \operatorname{supp} p+\operatorname{supp} q$. Choose $0<\lambda<\mu<1$ with $\lambda+\mu=1$ as above. By (4) and (5), we obtain that

$$
\begin{aligned}
-[\log \lambda \operatorname{tr} r p+\log \mu \operatorname{tr} r q] & =S(r \| \lambda p+\mu q)=S(\phi(r) \| \lambda \phi(p)+\mu \phi(q)) \\
& =-[\log \lambda \operatorname{tr} \phi(r) \phi(p)+\log \mu \operatorname{tr} \phi(r) \phi(q)] .
\end{aligned}
$$

We know that the rank-1 projections $\phi(p), \phi(q)$ are mutually orthogonal and

$$
\operatorname{supp} \phi(r) \subset \operatorname{supp} \phi(\lambda p+\mu q)=\operatorname{supp}(\lambda \phi(p)+\mu \phi(q))=\operatorname{supp} \phi(p)+\operatorname{supp} \phi(q) \text {. }
$$

It follows that $\operatorname{tr} \phi(r) \phi(p), \operatorname{tr} \phi(r) \phi(q)$ are non-negative real numbers with sum 1. Referring again to the easy fact that any element of a nontrivial compact real interval is a unique convex combination of the endpoints, we deduce from (6) that

$$
\operatorname{tr} \phi(r) \phi(p)=\operatorname{tr} r p
$$

This shows that when $\phi$ is restricted to the set of all rank-1 projections on $H$, it gives rise to a bijective transformation preserving the transition probability, i.e., a symmetry transformation. By Wigner's fundamental theorem, we infer that there is a unitary or antiunitary operator $U$ on $H$ such that $\phi(p)=U p U^{*}$ for every rank-1 projection $p$ on $H$. Now, consider the transformation $\psi: \rho \mapsto U^{*} \phi(\rho) U$. Clearly, this is a bijective map on $\mathcal{S}(H)$ which preserves the relative entropy and has the additional property that $\psi(p)=p$ holds for every rank-1 projection $p$. In what follows, we prove that $\psi(\rho)=\rho$ holds for every $\rho \in \mathcal{S}(H)$.

Let $\rho \in \mathcal{S}(H)$ be arbitrary. As $\psi$ preserves the relative entropy, similarly to (2), for any rank-1 projection $p$ on $H$, we have

$$
\operatorname{supp} p \subset \operatorname{supp} \rho \Leftrightarrow \operatorname{supp} p=\operatorname{supp} \psi(p) \subset \operatorname{supp} \psi(\rho),
$$

which gives us that $\operatorname{supp} \rho=\operatorname{supp} \psi(\rho)$. Moreover, for every such rank-1 projection $p$ on $H$, we have

$$
-\operatorname{tr} p \log \rho=S(p \| \rho)=S(\psi(p) \| \psi(\rho))=S(p \| \psi(\rho))=-\operatorname{tr} p \log \psi(\rho) .
$$

Let $p_{x}$ denote the projection onto the one-dimensional subspace of $H$ generated by the unit vector $x \in H$. For any unit vector $x \in \operatorname{supp} \rho=\operatorname{supp} \psi(\rho)$, inserting $p_{x}$ into equality (7), we see that

$$
\langle x|\log \rho| x\rangle=\langle x|\log \psi(\rho)| x\rangle .
$$

From this, we deduce that $\log \rho=\log \psi(\rho)$. This implies $\rho=\psi(\rho)=U^{*} \phi(\rho) U$ and hence we have $\phi(\rho)=U \rho U^{*}$, completing the proof of the theorem. 
The author was supported by the Hungarian National Foundation for Scientific Research (OTKA), Grant Nos. T046203 and NK68040, and by a joint Hungarian-Slovene grant.

${ }^{1}$ Bengtsson, I., and Życzkowski, K., Geometry of Quantum States: An Introduction to Quantum Entanglement (Cambridge University Press, Cambridge, 2006).

${ }^{2}$ Molnár, L., "Fidelity preserving maps on density operators," Rep. Math. Phys. 48, 299 (2001).

${ }^{3}$ Molnár, L., Selected Preserver Problems on Algebraic Structures of Linear Operators and on Function Spaces, Lecture Notes in Mathematics Vol. 1895 (Springer, Berlin, 2006), p. 236.

${ }^{4}$ Molnár, L., and Timmermann, W., "Isometries of quantum states," J. Phys. A 36, 267 (2003).

${ }^{5}$ Molnár, L., and Timmermann, W., "Preserving the measure of compatibility between quantum states," J. Math. Phys. 44, 969 (2003).

${ }^{6}$ Ohya, M., and Petz, D., Quantum Entropy and its Use (Springer-Verlag, Berlin, 1993).

${ }^{7}$ Schumacher, B., and Westmoreland, M. D., in Quantum Computation and Information, Contemporary Mathematics Vol. 305, edited by S. J. Lomonaco, Jr. and H. E. Brandt (American Mathematical Society, Providence, 2002), pp. 265-289.

${ }^{8}$ Vedral, V., "The role of relative entropy in quantum information theory," Rev. Mod. Phys. 74, 197 (2002). 